\title{
Damage Detection using Vibration Data and Dynamic Transmissibility Ensemble with Auto-Associative Neural Network
}

\author{
Yun-Lai ZHOU*, Magd Abdel WAHAB** \\ *Center for Offshore Research Engineering, National University of Singapore, Singapore \\ 2 Engineering Drive 2, Singapore, 117576 \\ **Division of Computational Mechanics, Ton Duc Thang University, Ho Chi Minh City, Vietnam \\ **Faculty of Civil Engineering, Ton Duc Thang University, Ho Chi Minh City, Vietnam \\ **Soete Laboratory, Faculty of Engineering and Architecture, Ghent University, Technologiepark Zwijnaarde 903, B-9052 \\ Zwijnaarde,Belgium, Email: magd.abdelwahab@tdt.edu.vn;magd.abdelwahab@ugent.be
}

cross $^{\text {ref }}$ http://dx.doi.org/10.5755/j01.mech.23.5.15339

\section{Introduction}

In the past decades, structural health monitoring $(S H M)$ has become a multidisciplinary research focus to the scientific communities. It attracted a lot of attention, due to the fact that engineering structures are commonly designed with more complexity and using more sophisticated newly invented material productions. Within daily use, the structures are usually and generally subjected to higher operational loads and unexpected loading conditions, and a demanding for longer lifecycle periods is required. Hence, numerous mechanical, civil and aerospace engineering researchers extensively developed vast of approaches for analysing the structural states that means to evaluate whether the structure is damaged or not, in order to prevent the anticipated damage, which might cause a vast loss in human daily lives in an inevitable way. Various categories of SHM procedures have been developed for analysing the structural states. Vibration-based, strain-based, Electrical Impedancebased, probability-based and statistical based methods have been studied and a quantity of papers, reports and books was published. Detailed literature review about the SHM can be found in [1-2].

Along with the development history of SHM, visual inspection approaches, such as penetrating liquids, is the commonest and most traditional and available technique, which will only be effective in those structures suitable for using liquids. Apart from this, taking advantage of the advancement of the science and technology, local on-line nondestructive $S H M$ becomes a main trend. And ultra-sound, lamb wave, eddy-current, $\mathrm{X}$ and Gamma rays, and laser measurement have been widely used in SHM. However, these advanced technologies normally require high experience that will largely decide on the quality of damage assessment.

Within all SHM technologies, the essential issue is to explore the interrelation between structural defects/deteriorations and those measured data. For this, conventional health monitoring and structural assessments have been evolved a large amount of outputs. SHM technologies can be divided into two directions, namely physical model based technologies and statistical model based methodologies. Physical model based technologies makes use of finite element analysis (FEA), regular experimental verification and further model updating is conducted in order to minimize the differences between the FEA and experimental measurements. Statistical model based methodologies concentrates on pattern recognition algorithms to discriminate the experimental measurements between the responses under intact condition and the responses under damaged condition. For a review on statistical model based techniques, the reader may refer to [1].

In $S H M$, the commonest way is to employ modal testing [3]. From modal testing and analysis, frequency response function $(F R F)$ are derived and applied to connect the structural excitation and dynamic responses. Therefore, $F R F$ can be utilized to detect structural defects and deteriorations [4-5]. In reference [4], curvature was applied to the $F R F$ measured at each location in the structure, and the change of $F R F$ curvature was used to detect structural damages with adopting a 10-degree-of-freedom lumped-mass system and I-40 bridge as verification, which demonstrated good manifestation in damage detection, localisation and quantification. In reference [5], a series of modal parameters based damage detection indicators were compared, i.e. mode shape, mode shape slope, mode shape curvature, mode shape curvature square and damage index. These five methods were extended to $F R F$ based damage detection analysis, and a free-free beam was simulated and dynamically tested to validate the damage detection methods. It was found that curvature based methods outperformed.

However, in real engineering applications, the measurement of excitation could be a big problem as loadings applied to the studied object are generally quite complicated. For instance, for a real bridge, apart from traffic loading, environmental uncertainties, such as boundary conditions, wind loading, temperature and humidity, still impose lots of influence on the dynamical behaviour of the bridge. In this case, avoiding the measurement of excitation, i.e. output based only methodologies would be a desirable pursue in SHM. Among all output-based methodologies, transmissibility, a concept raised decades ago, has become increasingly shared in scientific communities for its own merit depending on output only. Moreover, especially from the end of the $20^{\text {th }}$ century, booming research on transmissibility has been intensively developed [6-14].

Transmissibility has been put forward in a lot of applications such as force reconstruction, $F R F$ estimation and damage identification with referable reviews [6-7]. In reference [6], a general overview on transmissibility was given, and reviews of the generalization of transmissibility concept, virtues and restrictions were provided. In reference [7], force identification from transmissibility (in displace- 
ments and force) was given. However, this review concentrates on the application of transmissibility in identifying forces, i.e. the application of transmissibility. A recent and more general review on transmissibility history and applications was conducted in [2], where transmissibility was reviewed from both theoretical development and potential applications points of view. For a general definition and generalization for transmissibility, the reader may refer to [8].

In $S H M$, transmissibility has been used directly [9], or incorporated with other approaches, such as neural networks [10], discriminant analysis [11] and so on. For instance, in reference [9], transmissibility's measured along the structure were taken into consideration with the modal assurance criterion $(M A C)$ and then damage sensitive indicators were constructed. However, this approach did not separate the noise-contaminated frequency domain, i.e. the noise was also taken into account in the evaluation process. For joint working with other approaches, artificial neural networks are used in pattern recognition with using transmissibility as feature [10]. Mahalanobis' distance was used to reduce the noise influence in transmissibility estimation [11]. In addition, transmissibility has also been employed in operational modal analysis [12], force reconstruction [13], FRF estimation [14] and so on.

As cited above, even transmissibility based damage identification has progressed with lots of outputs, the early stage damage detection is still an open question. This is because problems still exist in real engineering applications when using SHM. For instance, how to analyse the big data captured nowadays in real engineering structures for intact and damaged states. Similar questions can be raised concerning the adaptability of methodologies in SHM, especially in industrial applications.

Normally transmissibility based damage detection procedures try to seek damage sensitive indicators that directly serve as measures to separate the structural patterns into damaged and undamaged ones. However, when the difference is small, it will be necessary to find a methodology to amplify the changes before and after damages. Among the entire machine learning algorithms, auto-associative neural network $(A A N N)$, raised decades ago [15-16], is a widely spread and effective tool for damage detection [1718]. The key idea of $A A N N$ is to learn the interrelation between structural responses and damages, and later to use the learning capacity to evaluate the new structural pattern, when outlier appears, or even damage occurs.

During last decades, $A A N N$ has been widely used in $S H M$ [17-25], and it has also been applied in driver operation modelling [26], speaker verification [27] and so on [28]. For application in $S H M$ [17-18], AANN is incorporated with other machine learning algorithms, such as factor analysis and principal component analysis, for detecting structural damages under environmental varieties. A three-story frame aluminium structure was experimented and used to draw out the applicability of the proposed damage detection procedure. It has been proved that $A A N N$ can successfully detect damages. In reference [19], $A A N N$ is adopted by comparing it with other four different methods: simple projection, principal component analysis, Sammon mapping, and outlier method in detecting local fault in a spur gear. It has been shown that $A A N N$ could provide much benefit in detecting damages. And this idea was repeated taking an Information Theoretic Criterion (AIC) into account in [20].
Later $A A N N$ was extended to damage detection for wind turbine blades [24-25]. In few years, $A A N N$ was adopted for analysing environmental and operational variability on $S H M$ [21]. Later, $A A N N$ was applied to identify potential faults in electric motors with combining wavelet transform [22]. Then, in reference [23], $A A N N$ are employed together with generalization techniques of information criterions and Bayesian regularization in order to construct three $A A N N$ models for detecting environmental-tolerant capacity, which was later verified by a cable-stayed bridge. And it has been proved that the $A A N N$ environmental tolerant capacity could be improved by early stopping technique, and $A A N N$ based $S H M$ uncertainty could be reduced with alarming threshold.

As discussed above, $A A N N$ proves to be effective in $S H M$, especially in damage detection. In this study, a new transmissibility-based technique using $A A N N$ is proposed in order to detect damage or novelty change. Considering the transmissibility method, vibration response and modal parameters will change due to the damage severity or novelty change. Afterwards, for each damage scenario, the transmissibility function of each node will be calculated and indicators to each node will be constructed, which are treated as input for $A A N N$. By setting a proper threshold, the proposed procedure can predict the new structural pattern related to outlier appearance or damage occurrence with a trained $A A N N$, which will function as a classifier in a later damage detection process. As for verification, a ten-floor numerical simulation is analysed in order to demonstrate the manifestation of the proposed method as well as unveil its applicability.

\section{Theoretical derivation}

\subsection{Transmissibility estimation}

In structural dynamics, for a linear multiple-degree-of-freedom $(M D O F)$ system, the dynamic equilibrium equation can be written by the well-known second order differential equation:

$$
M \ddot{x}(t)+C \ddot{x}(t)+K x(t)=f(t),
$$

where $M, C$ and $K$ are the mass, damping, and stiffness matrices of the system, respectively, $f(t)$ is the input force vector and $x(t)$ contains the responses of each DOF of the system.

Herein, for a harmonic applied force at a given coordinate, the transmissibility between point $i$ and a reference point $\mathrm{j}$ can be defined as:

$$
T_{(i, j)}=\frac{x_{i}}{x_{j}}
$$

where $X_{\mathrm{i}}$ and $X_{\mathrm{j}}$ are the complex amplitudes of the system responses $x_{i}(t)$ and $x_{j}(t)$, respectively.

Apart from direct calculating by the definition, transmissibility has kinds of ways in computation. For example, to use $F R F$ is a general method:

$$
T_{(i, j)}=\frac{x_{i}}{x_{j}}=\frac{H_{i k}}{H_{j k}},
$$


where $k$ is the excitation point, and $H$ represents the $F R F$. Apart from this, transmissibility can also be calculated by auto- and cross- spectrum of the responses, i.e.:

$$
T_{(i, j)}=\frac{x_{i}}{x_{j}}=\frac{G_{i k}}{G_{j k}}
$$

where $G$ means the auto- or cross-spectrum. Note here $k$ can equal to $i$ and $j$. Then when $k=i$ or $j$, auto-spectrum will appear. Herein, by choosing different reference points, it can be compared with the FRF estimation for avoiding the influence of noise, and then transmissibility coherence $(T C)$ can be derived. Detailed derivation of transmissibility is given in [6]. In this study, transmissibility is calculated by Equation (4).

\subsection{Auto-associative neural networks}

$A A N N$ is an effective pattern recognition, where damaged state or undamaged state could also be treated as a pattern. Decades ago, the conception of $A A N N$ was firstly addressed by Kramer [15-16]. The most important characteristic of $A A N N$ is that it could capture the interrelationship within a set of variables with embedding the correlation between those variables.

Commonly $A A N N$ is a five-layer perceptron feedforward network. Fig. 1 shows the Network architecture of the $A A N N$, where five typical layers, i.e. input layer, mapping layer, bottle neck layer, de-mapping layer, and output layer, are indicated. Note that the number of inputs and outputs is the same (in Figure 1, the input layer and output layer both have $s$ dimensions), which, thus, gives a possibility to use a low dimension of elements to characterize all the inputs, and finally gives an estimation of the structural state by using the difference between the inputs and outputs. Within $A A N N$ more hidden layers might be used to improve the performance of $A A N N$ in pattern recognition. Meanwhile, AANN could be considered as two independent threelayer neural networks connected in series, i.e. $B P$ neural networks. Herein, the first network compresses and mixes the $N$ redundant inputs into a smaller number of characteristic variables that should reveal the important and characteristic features. The second network performs in the opposite direction and regenerates the original $N$ redundant inputs data from the compressed information in the bottleneck layer.

In $S H M$, algorithms with $A A N N$ have numerous formulations, however, to all $A A N N$ based algorithms, the training data is all measured from the undamaged condition or operational condition without any damage or deterioration. The basic functions of $A A N N$ are the same functions as classifier for the data pattern from the baseline. The major difference between them is merely the input data that is the characteristic feature of the structure. Then, the testing data will be recognized from the data measured under undamaged condition. Herein, it is worth mentioning that one needs to bear in mind that the testing should be conducted under a normal condition, i.e. the condition should not impose a lot of influences to the structural measured responses compared with the measurements under undamaged condition.

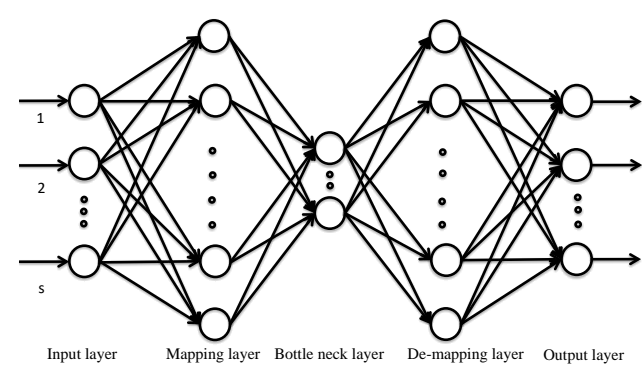

Fig. 1 Network architecture of the AANN

In AANN, one can construct a structural characteristic matrix $A$ that may contain $s$ dimensions as input for $A A N N$. Conventional SHM may use modal properties as characteristic matrix. Here, $A$ represents a general description of structural characteristic that can mean modal properties or any other modal parameters. However, in this study, it means the transmissibility based damage sensitive features. In Fig. 1, $A$ means the components in the input layer.

Herein, it is necessary to give a basic description of the $A A N N$ working procedure, namely (1) training the $A A N N$ and (2) predicting the new structure pattern. For the first stage, i.e. training stage, a specific number of damage scenarios should be used to train the $A A N N$ as to learn the capacity of recognizing the damaged scenarios from the undamaged scenarios. Then, to the predicting stage, new structural scenarios will be taken into account to test the trained $A A N N$, which will perform as a classifier. Note that the trained $A A N N$ might not perform well in the beginning after training that depends on the engineer experience. And attention also should be paid to the number of nodes in bottleneck layer, which should be well chosen. The number of nodes in bottleneck should be at least larger than the independent variables of all the characteristics. Otherwise, it will lose some characteristic information and imposes big influence in the later original data regeneration.

For testing matrix, $A$, the error between input and output can be described as:

$$
d=A-A_{\text {out }},
$$

where $A_{\text {out }}$ is the output of the $A A N N$ in output layer in Fig. 1 with input $A$ in input layer in Fig. 1, and $d$ means the error or damage indicator in our case. In order to estimate whether the structure is damaged or not, one needs to set a threshold for $d$ as tolerance, for instance, $1 \%$. Those beyond $1 \%$ will be considered as damaged. Herein, it is necessary to bear in mind that if a different error tolerance is chosen, the predicted results might show some damages in the structure, while under another threshold it is undamaged. And the error tolerance is chosen depending on engineer experience and it may be associated with the environmental uncertainties.

\section{Damage detection procedure}

\subsection{Inputs for $A A N N$}

The input parameters for $A A N N$ are the most essential part for the algorithm, which should reveal the structural dynamic characteristics. Since transmissibility has been discussed before in the aforementioned sections, herein, one indicator upon using transmissibility is developed in order 
to enhance the sensitivity of transmissibility associative to the structural deterioration or damages. The indicator takes the sum of transmissibility along the specific frequency range that can be described as:

$$
T I_{i}=\int_{f_{\min }}^{f_{\max }} T_{i, j} d f
$$

where $f_{\min }$ and $f_{\max }$ are the low and high boundary for the integration area. A clear demonstration for this is shown in Fig. 2.

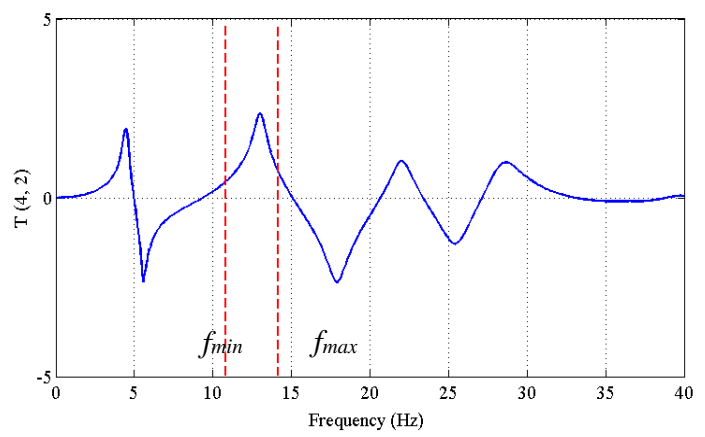

Fig. 2 A description for the frequency band

\subsection{Damage detection indicator}

Damage detection experienced a lot of advancements during last years, and in order to show the feasibility of the proposed damage detection procedure, a comparison between its performance and previous research outputs is presented in this section. In references [29-32], different damage detection methods are illustrated. For instance, in [29], building stability monitoring and diagnostic procedure is described with taking frequency change to detect damages; in [30], a norm employing the structural properties and excitation are constructed and used for identifying structural damages; in [31], time-frequency analysis is discussed to draw out the damage detection procedure with using power spectrum density $(P S D)$; in [32], continuous wavelet transform $(C W T)$ is utilized to analyze the acceleration and velocity response to detect the damages. In this study, the frequency change is taken into consideration as comparison for the proposed damage detection procedure, which is indicated as:

$$
D F_{i}=f_{i}^{d}-f_{i}^{u}
$$

where $D F_{i}$ means the $i^{\text {th }}$ modal frequency difference between undamaged state and damaged state. And $f_{i}$ mean $i^{\text {th }}$ modal frequency, ()$^{d}$ and ()$^{u}$ mean the value under damaged state and undamaged state, respectively.

\subsection{Damage detection flowchart}

As for damage detection procedure, those detailed steps can be illustrated as follows:

Step 1: Transmissibility evaluation. Transmissibility is estimated from Eq. (4) for all measurements.

Step 2: Transmissibility selection. This step is complicated. It is challenging to select transmissibility for lateral analysis, while one possibility is to do transmissibility selection with dimension reduction approaches. In this study, transmissibility is selected by engineering experience, which will largely affect the final results.

Step 3: Feature extraction. Transmissibility based damage feature is derived from Eq. (6), which will be finally taken as inputs for $A A N N$ in training and testing.

Step 4: $A A N N$ training and testing. The derived features are divided into two parts, i.e. one part is taken for $A A N N$ training, and another part is used for testing.

Step 5: Damage indicator calculation. For comparison reason, frequency change for each damage state is calculated referring to the undamaged state.

Step 6: Results analysis. To confirm whether the predicted results are satisfactory or not using the engineering experience, if not, to re-select the transmissibility as step 2 might hold great influence in the predicted results. If the results are satisfactory, then the analysis is completed.

\section{Numerical verification}

\subsection{Model description}

For checking the feasibility of the proposed damage detection procedure, herein, a simulation of ten-floor structure is employed as shown in Fig. 3. The masses and stiffness's are: $m_{1}=m_{2}=\ldots=m_{10}=1 \mathrm{~kg}$ and $k_{1}=k_{2}=k_{3}=$ $\ldots=k_{10}=10^{4} \mathrm{~N} / \mathrm{m}$.

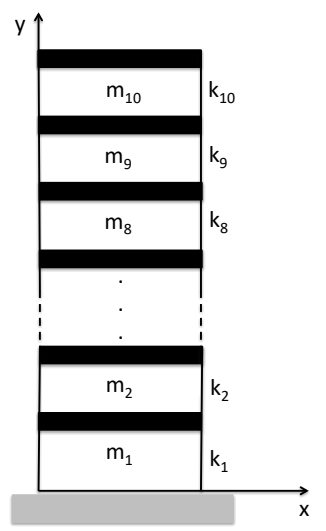

Fig. 3 A schematic diagram of the ten-floor model

Note that damage model in this study is simulated as stiffness reduction, which is a common model that can be found in SHM analysis. Both single and multiple damages are numerically analysed. For single damage, two categories are simulated where stiffness reduction is introduced to $\mathrm{k}_{3}$ and $\mathrm{k}_{7}$, separately. For each of them, a stiffness reduction from $0 \%$ to $50 \%$ with an interval of $0.5 \%$ is recorded. Then, for each single damage in $\mathrm{k}_{3}$ and $\mathrm{k}_{7}, 100$ scenarios are considered. Therefore, including undamaged scenario, there will be a total of 201 scenarios. And 100 of those damaged scenarios together with undamaged scenario (in total 101 scenarios) are taken for AANN training; and the other 100 damaged scenarios together with undamaged scenario (in total 101 scenarios) are taken for AANN testing and predicting. For multiple damaged scenarios, damage is introduced to $\mathrm{k}_{3}$ and $\mathrm{k}_{7}$ at the same time, and a stiffness reduction from $0 \%-50 \%$ with interval $0.25 \%$ is recorded. Thus, there will be again 201 scenarios including undamaged scenario. Same as before, 100 damaged scenarios together with undamaged scenario (in total 101 scenarios) will be taken for 
AANN training, and the other 100 damaged scenarios together with undamaged scenario (in total 101 scenarios) will be taken for AANN testing and predicting. In addition, in order to account for the environmental variability, $2 \%$ random noise is taken into consideration for the previous damaged scenarios analysis.

\subsection{Results discussion}

This section will give a discussion of the calculated results of the proposed damage detection procedure.

\subsubsection{Noisy free scenarios}

Fig. 4 shows the error percentage of training results for single damage where in total 101 scenarios are taken into account. Note that the first scenario is undamaged, second scenario to $51^{\text {st }}$ scenario for damage introduced into the third floor, and scenario $52^{\text {nd }}$ to $101^{\text {st }}$ are the scenarios for damage introduced to the seventh floor.

From Fig. 4, one can find that the error is under a very limit range, i.e. the maximum is under $0.5 \%$, which means that the AANN is well trained and well-constructed.

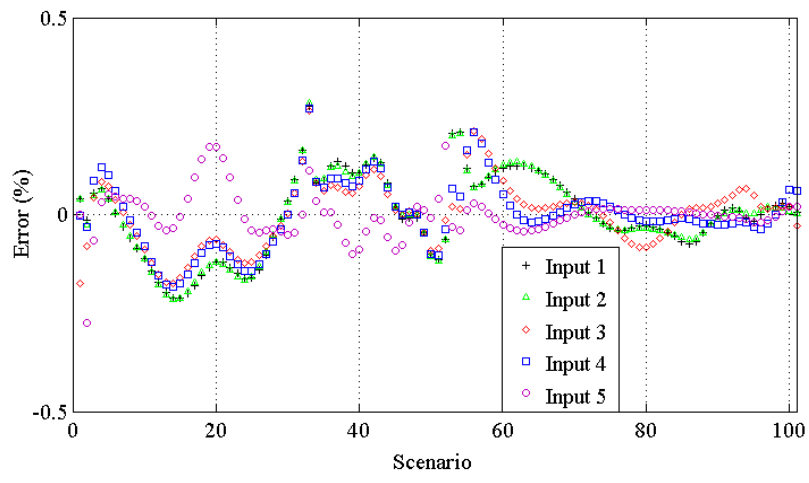

Fig. 4 Error of trained AANN for 101 scenarios

Fig. 5 shows the predicted results, the damage indicator, ' $d(\%)$ ', for the single damage in $\mathrm{k}_{3}$ of 50 scenarios, as well as, undamaged scenario. Note that first scenario means undamaged scenario, second scenario to $51^{\text {th }}$ scenario are the 50 damaged scenarios for single damage in $\mathrm{k}_{3}$. From Fig. 5, one can find that all the fifty damage scenarios are successfully detected, as one can find clear difference between intact scenario and damaged scenarios. Besides, it can be also found that ' $\mathrm{d}(\%)$ ' of input 3, 4, and 5 enlarge monotonically as the damage severity increases, which might suggest that ' $d(\%)$ ' is able to quantify the damage severity. Fig. 6 demonstrates the predicted results of damage indicator for single damage in $\mathrm{k}_{7}$ of 50 scenarios, as well as, undamaged scenario. Similar to Fig. 5, in Fig. 6 all the fifty damaged scenarios are detected successfully. However, for small damage severities, it is necessary to zoom in the figure to get a clear observation. Comparing Fig. 6 with Fig. 5, one can find that the damage indicator of Fig. 6 changes much less than that in Fig. 5. This is caused by the input selection, i.e. different inputs will have different interrelation with $\mathrm{k}_{3}$ and $\mathrm{k}_{7}$, respectively. And this will finally determine the predicted results.

Fig. 7 shows the predicted results for multiple damages scenario, i.e. damage in $k_{3}$ and $k_{7}$ with 100 damage scenarios, as well as, undamaged scenario. The first scenario is intact, and second scenario to $101^{\text {th }}$ scenario are damaged scenarios. From Fig. 7, it can be seen that all the damaged scenarios are separated from the undamaged scenario, while the damage indicator of second scenario varies slightly compared to that of other damaged scenarios. Herein, it can be concluded that if AANN and inputs are well chosen, the algorithm is capable of detecting damages.

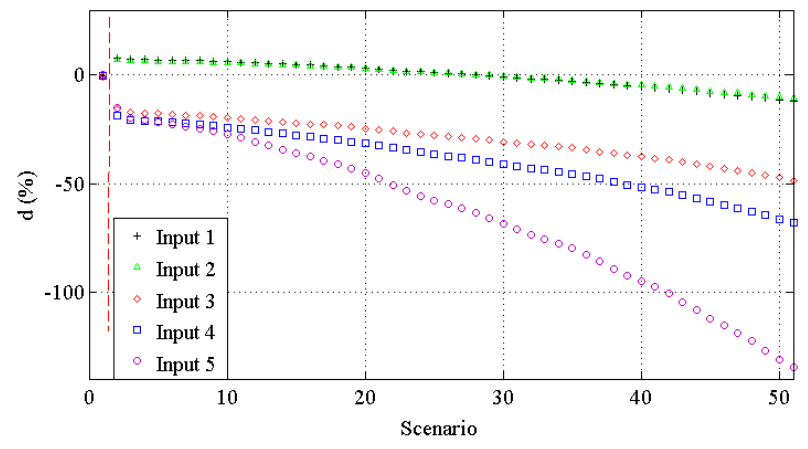

Fig. 5 Prediction of AANN for the single damage in $\mathrm{k}_{3}$ for 50 damaged scenarios with intact scenario

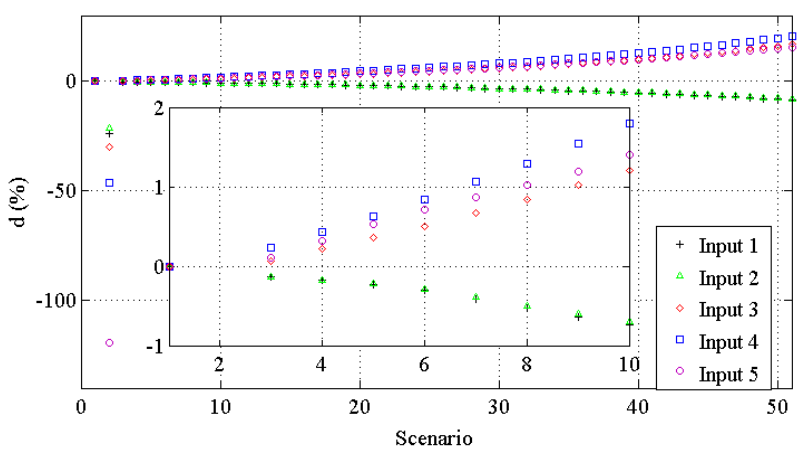

Fig. 6 Prediction of AANN for the single damage in $\mathrm{k}_{7}$ for 50 damaged scenarios with intact scenario

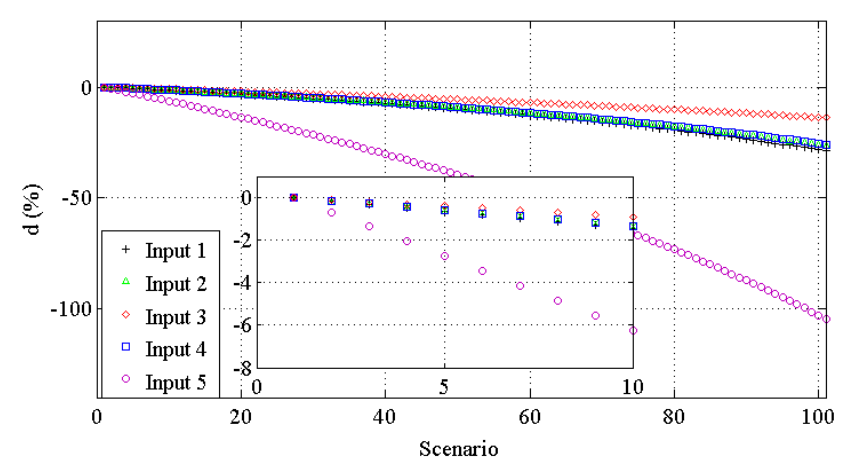

Fig. 7 Prediction of AANN for the multiple damages in $k_{3}$ and $k_{7}$ for 100 damaged scenarios with intact scenario

Figs. 8 and 9 show the results of $D F_{1}$ for the single damage scenarios and multiple damage scenarios. And from Figs. 8 and 9, one can find that all the damaged scenarios are successfully identified from the undamaged scenario (Scenario 1). And comparing these two figures with Figs. 5, 6 , and 7 , both methods show feasibility in detecting damages. 


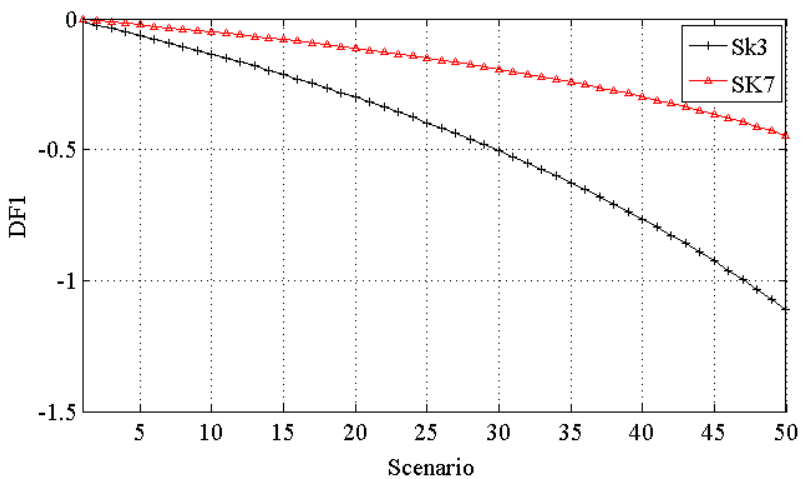

Fig. $8 D F_{1}$ for single damage in $\mathrm{K} 3$ and $\mathrm{K} 7$

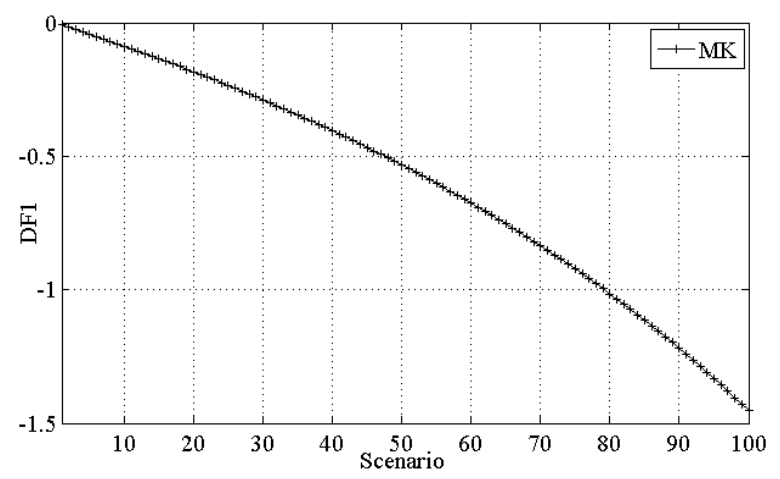

Fig. $9 D F_{1}$ for multiple damages in $\mathrm{K} 3$ and $\mathrm{K} 7$

\subsubsection{Noisy scenarios}

Figs. 10 and 11 show the damage indicator for single damage in $\mathrm{k}_{3}$ and $\mathrm{k}_{7}$ with $2 \%$ random noise for 50 damaged scenarios, as well as, undamaged scenario, respectively. From Figs. 10 and 11, it can be seen that all damage scenarios can be successfully detected, while for small damage severities, the damage indicator varies slightly, which requires amplification for better illustration of the differences. Same as noise free scenarios, the damage indicator in Fig. 10 varies much more than that in Fig. 11. The reason behind this is the same as described before, i.e. different input selection will affect the final performance as they have different interrelation with $\mathrm{k}_{3}$ and $\mathrm{k}_{7}$, respectively. Herein, comparing Figs. 10 and 11, with Figs. 5 and 6, one can find that random noise imposes influences to the final results, and the small damage severities become challenging to detect.

Fig. 12 demonstrates the predicted results for multiple damages in $k_{3}$ and $k_{7}$ with $2 \%$ random noise for 100 damaged scenarios with intact scenario. From Fig. 12, it can be seen that most of the damaged scenarios can be detected while for small damage scenario, it will be challenging to detect. Only the damage indicator of input 5 gives a clear difference compared with that of undamaged scenario. Comparing Fig. 12 with Fig. 7, it can be found that noise did not impose as much influence to the single damage as to multiple damages. This means that damage severity plays more essential role than random noise in the final results contribution.

Figs. 13 and 14 show the results of $D F_{1}$ for the single damage scenarios and multiple damage scenarios with 2\% noise. And from Figs. 13 and 14, all the damaged scenarios are successfully identified from the undamaged

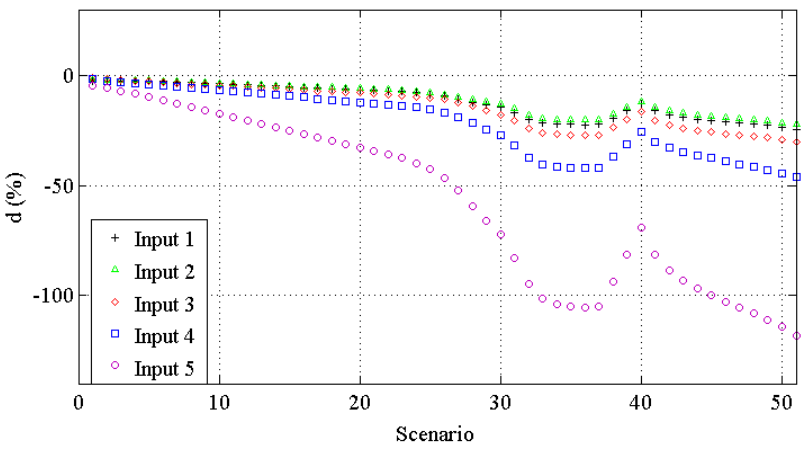

Fig. 10 Prediction of AANN for the single damage in K3 for 50 damaged scenarios with intact scenario with $2 \%$ random noise

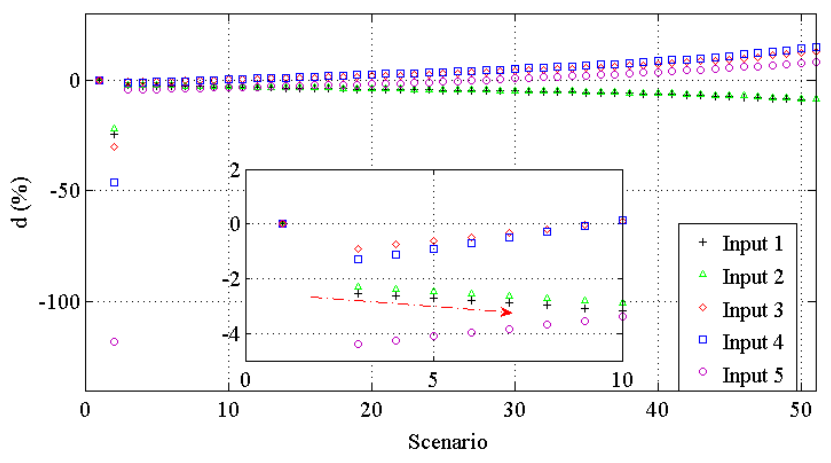

Fig. 11 Prediction of AANN for the single damage in K7 for 50 damaged scenarios with intact scenario with $2 \%$ random noise

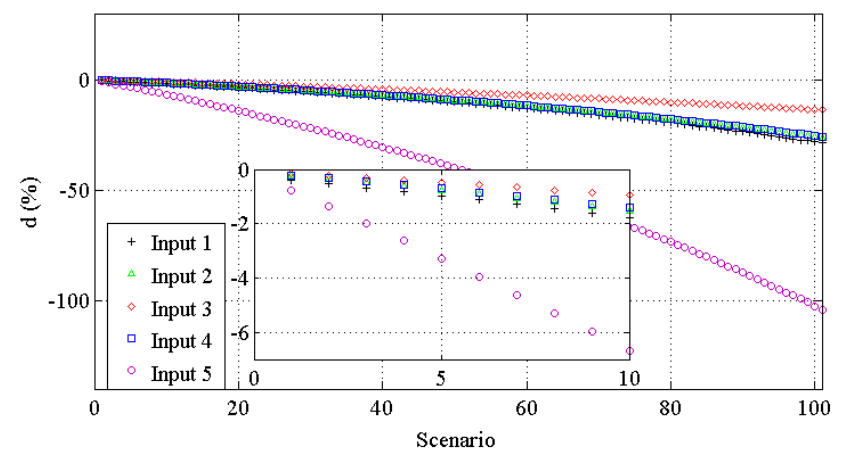

Fig. 12 AANN predicted for the multiple damages in $\mathrm{K} 3$ and $\mathrm{K} 7$ for 100 damaged scenarios with intact scenario with $2 \%$ random noise

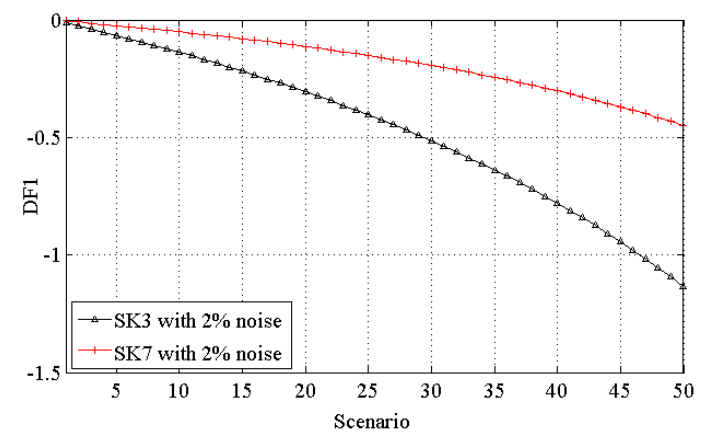

Fig. $13 D F_{1}$ for single damage in $\mathrm{K} 3$ and $\mathrm{K} 7$ with $2 \%$ noise

scenario (Scenario 1). And comparing these two figures with Figs. 10, 11, and 12, both methods show feasibility in detecting damages. 


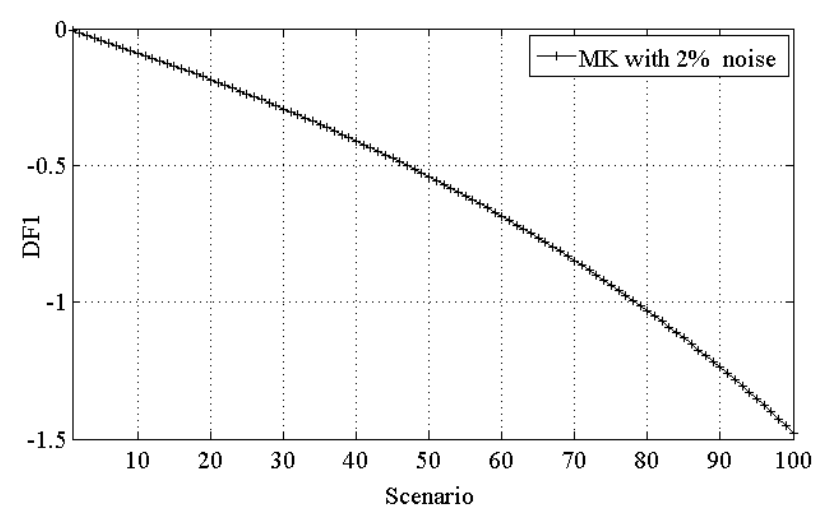

Fig. $14 D F_{1}$ for multiple damages in $\mathrm{K} 3$ and $\mathrm{K} 7$ with $2 \%$ noise

\section{Conclusions}

This study illustrated a damage detection procedure using transmissibility together with $A A N N$. Unlike conventional $S H M$ techniques, this damage detection procedure only relies on the structural dynamic responses, and the use of $A A N N$ makes it possible to detect damages once the baseline is defined. The advantage of this study is that it newly introduced the implementation of $A A N N$ into transmissibility based damage detection procedure. This procedure is validated using a ten-floor simulated structure taking into account random noise. The results showed promising potential extension in further application of the proposed technique. Note that $A A N N$ and inputs for $A A N N$ should be well chosen. This can largely determine the capacity of damage detection, and further investigation should also be conducted for robustness enhancement.

\section{Acknowledgements}

The first author thanks CWO (Commissie Wetenschappelijk Onderzoek), Faculty of Engineering and Architecture, Ghent University for providing financial support for a research stay at Soete Laboratory.

\section{References}

1. Sohn, H.; Farrar, C.R.; Hemez, F.M.; Shunk, D.D.; Stinemates, D.W.; Nadler, B.R.; Czarnecki, J.J. 2004, A Review of Structural 1996-2001. Los Alamos National Laboratory Report, LA-13976-MS, 2004.

2. Doebling, S.W.; Farrar, C.R.; Prime, M.B.; Shevitz, D.W. 1996. Damage Identification and Health Monitoring of Structural and Mechanical Systems from Changes in Their Vibration Characteristics, A Literature Review, Los Alamos National Laboratory Report, LA-13070MS, 1996.

3. Maia, N.M.M.; Silva, J.M.M. 2003. Theoretical and Experimental Modal Analysis (Mechanical Engineering Research Studies: Engineering Dynamics), Editor: Research Studies Press (8 January 2003), Colección: Mechanical Engineering Research Studies: Engineering Dynamics, ISBN-10: 0863802087, ISBN-13: 9780863802089.

4. Sampaio, R.P.C., Maia, N.M.M., Silva, J. 1999. Damage detection using the frequency-response-function curvature method, Journal of Sound and vibration 226
(5): 1029-1042.

http://dx.doi.org/10.1006/jsvi.1999.2340.

5. Maia, N.M.M., Silva, J.M.M., Almas, E.A.M. 2003. Damage detection in structures: from mode shape to frequency response function methods, Mechanical Systems and Signal Processing 17(3): 489-498.

http://dx.doi.org/10.1006/mssp.2002.1506.

6. Maia, N.M.M., Urgueira, A.P.V., Almeida, R.A.B. 2011. Whys and Wherefores of Transmissibility. Vibration Analysis and Control - New Trends and Developments, edited by Francisco Beltran-Carbajal, ISBN 978953-307-433-7, 364 pages, Publisher: InTech, Chapters published September 06, 2011. http://dx.doi.org/ $10.5772 / 24869$.

7. Maia, N.M.M., Lage, Y.E., Neves, M.M. 2011. Chapter 6: Recent Advances on Force Identification in Structural Dynamics. Book: Advances in Vibration Engineering and Structural Dynamics, Edited by Francisco Beltran-Carbajal, ISBN 978-953-51-0845-0, 378 pages. Publisher: InTech, pp.103-132. http://dx.doi.org/ 10.5772/51650.

8. Ribeiro, A.M.R., Silva, J.M.M., Maia, N.M.M. 2000. On the generalization of the transmissibility concept, Mechanical Systems and Signal Processing 14 (1): 2935.

http://dx.doi.org/ 10.1006/mssp.1999.1268.

9. Maia, N.M.M., Almeida, R., Urgueira, A., Sampaio, R. 2011. Damage detection and quantification using transmissibility, Mechanical Systems and Signal Processing 25: 2475-2483. http://dx.doi.org/10.1016/j.ymssp.2011.04.002.

10. Chen, Q., Chan, Y.W., Worden, K., Tomlinson, G.R. 1994. Structural fault detection using neural networks trained on transmissibility functions, In: Proceedings of the International Conference on Vibration Engineering, Beijing, China, 1994, p.p. 456-646.

11. Worden, K., Manson, G., Fieller, N.R.J. 2000. Damage detection using outlier analysis, Journal of Sound and Vibration 229(3): 647-667.

http://dx.doi.org/ 10.1006/jsvi.1999.2514.

12. Yang, W.J., Ren, W.X. 2012. Operational Modal Parameter Identification from Power Spectrum Density Transmissibility, Computer-Aided Civil and Infrastructure Engineering 27(3): 202-217. http://dx.doi.org/10.1111/j.1467-8667.2011.00735.x.

13. Lage, Y.E., Maia, N.M.M., Neves, M.M. 2014. Force Magnitude Reconstruction Using the Force Transmissibility Concept, Shock and Vibration ID 905912. http://dx.doi.org/10.1155/2014/905912.

14. Urgueira, A.P.V., Almeida, R.A.B., Maia, N.M.M. 2011. On the use of the transmissibility concept for the evaluation of frequency response functions, Mechanical Systems and Signal Processing 25: 940-951. http://dx.doi.org/10.1016/j.ymssp.2010.07.015.

15. Kramer, M.A. 1991. Nonlinear principle component analysis using auto-associative șepineural networks, AIChE Journal (37) 2: 233-243. http://dx.doi.org/10.1002/aic.690370209.

16. Kramer, M.A. 1992. Auto-associative neural networks, Computers in Chemical iscepiengineering (16) 4: 313-328. http://dx.doi.org/10.1016/0098-1354(92)80051-A.

17. Figueiredo, E. 2010. Damage Identification in Civil Engineering Infrastructure under Operational and Environmental Conditions, $\mathrm{PhD}$ thesis. 
18. Figueiredo, E.; Park, G.; Farrar, C.R.; Worden, K.; Figueiras, J. 2010. Machine learning algorithms for damage detection under operational and environmental variability, Structural Health Monitoring 0(0): 1-14. http://dx.doi.org/10.1177/1475921710388971.

19. Worden, K.; Manson, G. 1999. Visualization and Dimension Reduction of High-dimensional Data for Damage Detection, Proceedings of IMAC XVII - 17th International Modal Analysis Conference. Kissimmee, Florida, USA. February 8-11, 1999.

20. Sohn, H.; Worden, K.; Farrar, C.R. 2001.Novelty detection using auto-associative neural networks, Proceedings of 2001 ASME International Mechanical Engineering Congress and Exposition. November 11-16, 2001, New York.

21. Sohn, H. 2007. Effects of environmental and operational variability on structural health monitoring, Philosophical Transactions of the Royal Society A 365: 539560.

http://dx.doi.org/10.1098/rsta.2006.1935.

22. Gullulu, S.; Seker, S. 2011. Signal based approach for data mining in fault detection of induction motor, Scientific Research and Essays 6(22): 4720-4731. http://dx.doi.org/10.5897/SRE11.817.

23. Zhou H.F.; Ni, Y.Q.; Ko, J.M. 2011. Structural damage alarming using auto-associative neural network technique: Exploration of environment-tolerant capacity and setup of alarming threshold, Mechanical Systems and Signal Processing 25: 1508-1526. http://dx.doi.org/10.1016/j.ymssp.2011.01.005.

24. Dervilis, N.; Barthorpe, R.; Antoniadou, I.; Worden, K. 2012. Impact Damage Detection for Composite Material Typical of Wind Turbine Blades Using Novelty Detection Proceedings of 6th European Workshop on Structural Health Monitoring, Dresden, Germany, July 2012.

25. Yang, H.-H.; Huang M.-L.; Yang S.-W. 2015. Integrating Auto-Associative Neural Networks with Hotelling $\mathrm{T}^{2}$ Control Charts for Wind Turbine Fault Detection, Energies 8: 12100-12115. http://dx.doi.org/10.3390/en81012100.

26. Othman, MDR.; Zhang, Z.; Imamura T.; Miyake, T. 2009. Modeling Driver Operation Behavior by Linear Prediction Analysis and Auto Associative Neural Network, Proceedings of the 2009 IEEE International Conference on Systems, Man, and Cybernetics, San Antonio, TX, USA - October 2009. https://doi.org/10.1109/ICSMC.2009.5346668.

27. Garimella, S.; Hermansky, H. 2013. Factor Analysis of Auto-Associative Neural Networks with Application in Speaker Verification. IEEE Transactions On Neural Networks and Learning Systems, 24 (4), April 2013.

28. Sangeetha, J.; Jothilakshmi, S. 2015. A novel spoken document retrieval system using AutoAssociative Neural Network based keyword spotting, IEEE Sponsored 9th International Conference on Intelligent Systems and Control (ISCO) 2015.

29. Volkovas, V. 2013. The concept of buildings stability monitoring and damage diagnostics, Key engineering materials (569-570): 238-245.

http://dx.doi.org/10.4028/www.scientific.net/KEM.569-570.238.

30. Petkevičius, K.; Volkovas, V. 2011. Monitoring and identification of structural damages damages, Mechanika 17(3): 246-250. http://dx.doi.org/10.5755/j01.mech.17.3.498.

31. Volkovas, V.; Petkevičius, K.; Eidukevičiūtè, M.; Akinci, TC. 2012. Diagnostics of construction defects in a building by using time-frequency analysis, Mechanika 18(4): 432-437. http://dx.doi.org /10.5755/j01.mech.18.4.2340.

32. Volkovas, V.; Eidukevičiūtè, M.; Nogay, H.S.; Akinci, T.C. 2012. Application of wavelet transform to defect detection of building's structure, Mechanika 18(6): 683-690. http://dx.doi.org/10.5755/j01.mech.18.6.3169.

Yun-Lai Zhou, Magd Abdel Wahab

\section{DAMAGE DETECTION USING VIBRATION DATA AND DYNAMIC TRANSMISSIBILITY ENSEMBLE WITH AUTO-ASSOCIATIVE NEURAL NETWORK}

S u m m a r y

In this paper, a transmissibility based damage detection methodology using artificial intelligence is proposed. Structural health monitoring requires accurate damage detection in real engineering while the environmental uncertainties make this a challenge. In order to reduce this effect, artificial intelligence, such as artificial neural networks might be a possible strategy for achieving a better interpretation of the monitored data during operational condition. In this study, transmissibility is taken into account as damage sensitive feature because it accounts for the response data only. Then, auto-associative neural network is employed for detecting the structural damage and predicting its severity. In order to validate our proposed technique, a ten-floor structure is simulated and studied. The results show good performance in detecting damages.

Keywords: transmissibility; damage detection; auto-associative neural network

Received June 30, 2016

Accepted October 13, 2017 